\title{
Six-years survival and predictors of mortality after CABG using cold vs. warm blood cardioplegia in elective and emergent settings
}

Mohamed Zeriouh ${ }^{1 *}$, Ammar Heider ${ }^{1 \dagger}$, Parwis B. Rahmanian ${ }^{1 \dagger}$, Yeong-Hoon Choi ${ }^{1}$, Anton Sabashnikov ${ }^{1}$, Maximillian Scherner ${ }^{1}$, Aron-Frederik Popov ${ }^{2}$, Alexander Weymann ${ }^{3}$, Ali Ghodsizad ${ }^{4}$, Antje-Christin Deppe ${ }^{1}$ Axel Kröner ${ }^{1}$, Ferdinand Kuhn-Régnier ${ }^{1}$, Jens Wippermann ${ }^{1}$ and Thorsten Wahlers ${ }^{1}$

\begin{abstract}
Background: The aim of this study was to determine whether intermittent warm blood cardioplegia (IWC) is associated with comparable myocardial protection compared to cold blood cardioplegia (ICC) in patients undergoing elective vs. emergent CABG procedures.
\end{abstract}

Methods: Out of 2292 consecutive patients who underwent isolated on-pump CABG surgery using cardioplegic arrest either with ICC or IWC between January 2008 and December 2010, 247 consecutive emergent patients were identified and consecutively matched 1:2 with elective patients based on gender, age ( $<50$ years, 50-70 years, $>70$ years) and ejection fraction $(<40 \%, 40-50 \%,>50 \%)$. Perioperative outcomes and long-term mortality were compared between ICC and IWC strategies and predictors for 30-day mortality and perioperative myocardial injury were identified in both elective and emergent subgroups of patients.

Results: Preoperative demographics and baseline characteristics, logistic Euroscore, CPB-time, number of distal anastomoses and LIMA-use were comparable. Aortic cross clamp time was significantly longer in the IWC-group regardless of the urgency of the procedure ( $p=0.05$ and $p=0.015$ for emergent and elective settings). There were no significant differences regarding ICU-stay, ventilation time, total blood loss and need for dialysis. The overall 30-day, 1-, 3- and 6-year survival of the entire patient cohort was 93.7, 91.8, 90.4 and $89.1 \%$, respectively, with significantly better outcomes when operated electively $(p<0.001)$ but no differences between ICC and IWC both in elective ( $p=0.857$ ) and emergent $(p=0.741)$ subgroups. Multivariate analysis did not identify the type of cardioplegia as a predictor for 30-day mortality and for perioperative myocardial injury. However, independent factors predictive of 30-day mortality were: EF < 40 \% (OR 3.66; 95 \% Cl: 1.79-7.52; $p<0.001$ ), atrial fibrillation (OR 3.33; 95 \% Cl: 1.49-7.47; $p<0.003$ ), peripheral artery disease (OR 2.51; $95 \%$ Cl: 1.13-5.55; $p<0.023)$ and COPD (OR 0.26; $95 \%$ Cl: 1.05-6.21; $p<0.038$ ); predictors for perioperative myocardial infarction were $\mathrm{EF}<40 \%$ (OR 2.04; $95 \% \mathrm{Cl}: 1.32-3.15 ; p<0.001$ ), preoperative IABP support (OR 3.68; 95 \% Cl: 1.34-10.13; $p<0.012$ ), and hemofiltration (OR 3.61; $95 \%$ Cl: 2.22-5.87; $p<0.001$.

Conclusion: Although the aortic cross clamp time was prolonged in the IWC group our results confirm effective myocardial protection under IWC, regardless of the urgency of the procedure. We suggest that intermittent warm cardioplegia in emergent CABG setting is a low-cost alternative and safe. It is associated with similar long-term outcomes both in elective and emergent settings compared to intermittent cold cardioplegia.

\footnotetext{
* Correspondence: mohamed.zeriouh@uk-koeln.de

${ }^{\dagger}$ Equal contributors

'Department of Cardiothoracic Surgery, Heart Center, University of Cologne, Kerpener Str. 62, 50937 Cologne, Germany

Full list of author information is available at the end of the article
}

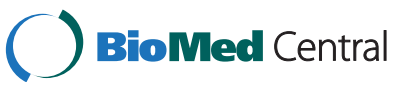

(c) 2015 Zeriouh et al. Open Access This article is distributed under the terms of the Creative Commons Attribution 4.0 International License (http://creativecommons.org/licenses/by/4.0/), which permits unrestricted use, distribution, and reproduction in any medium, provided you give appropriate credit to the original author(s) and the source, provide a link to the Creative Commons license, and indicate if changes were made. The Creative Commons Public Domain Dedication waiver (http://creativecommons.org/publicdomain/zero/1.0/) applies to the data made available in this article, unless otherwise stated. 


\section{Background}

The concept of myocardial protection during cardiac surgery have been already described in detail in the 1950s by Bigelow, who proposed to use hypothermia as a strategy to protect the myocardium in heart surgery [1]. In this respect, a reduction in myocardial oxygen consumption up to $80 \%$ can be achieved by lowering the blood temperature up to $20{ }^{\circ} \mathrm{C}$. However, the disadvantage of the isolated hypothermia without an additional usage of cardioplegia results in an incomplete electromechanical cardiac arrest with ventricular fibrillation. Therefore, subsequent studies by Gay and Ebert support the use of potassium-rich solution for the induction of diastolic cardiac arrest by depolarization [2]. In addition, further development of blood cardioplegia by Follette and Buckberg as a composition of blood and crystalloid solution improved myocardial protection enormously [2, 3]. Finally, the next breakthrough was the development of normothermic blood cardioplegia in which Calafiore has set new standards in myocardial protection during cardiac surgery $[4,5]$.

Currently, a large variety of techniques for myocardial protection is available in cardiac surgery. Most surgical centers in the USA and west european countries preferably apply blood cardioplegia as several studies indicated superiority of blood cardioplegia over crystalloid solutions [6]. Nevertheless, the debate over the optimal cardioplegia strategy, the way of application and temperature is still under discussion [7]. Moreover, other additional potentially cardioprotective effects on ischemic myocardium are discussed in the literature [8]. In our institution intermittent blood cardioplegia is used in coronary artery bypass grafting (CABG) patients and is administered antegradely either as intermittent normothermic cardioplegia (IWC) according to Calafiore's warm blood cardioplegia or intermittent hypothermic cardioplegia (ICC) according to Buckberg's cold blood cardioplegia. Nowadays, the IWC as a simple and costeffective method is in widespread use throughout the world. Whereas several studies have been focused on differences and similarities in blood cardioplegia in elective CABG patients, there has been a lack of evidence in terms of applicability of different cardioplegia in emergent settings. The aim of the present study was to critically examine both myocardial protection strategies in patients undergoing emergent CABG and patients receiving elective $\mathrm{CABG}$.

\section{Methods}

\section{Patients}

This was a retrospective study at the Heart Center of the University of Cologne with a cohort consisting of a total of 2292 patients who underwent isolated CABG from January 2008 to December 2010. Of them 247 consecutive patients were treated as an emergency and were consecutively matched 1:2 with elective coronary bypass patients based on age $(<50,50-70$ or $>70$ years), gender and ejection fraction $(<40 \%, 40-50 \%$ or $>50 \%)$. Due to several missing values in terms of preoperative patient characteristics and differences regarding baseline data between elective and emergent patients, the number of elective patients with exact matching criteria reached 448. In total, we explored two groups with a total number of 695 patients, subsequently dividing them depending on the blood cardioplegia strategy, with 176 patients $(25.3 \%)$ receiving ICC versus 506 patients $(72.8 \%)$ receiving IWC. A total of 13 patients were excluded after matching due to missing variables of interest. A subgroup analysis for each urgency status was performed. Furthermore, factors associated with early mortality as well as independent factors predictive of 30-day mortality in elective and emergent settings were evaluated.

\section{Operation technique}

CABG was standardized in terms of surgical technique and perioperative management. In brief, all operations were performed with cardiopulmonary bypass $(\mathrm{CPB})$ via median sternotomy. CPB was implemented with arterial cannulation of the ascending aorta and venous drainage which was connected to the right atrium with a twostage venous cannula. A root suction was always placed in the aortic root. Left and/or right internal mammary arteries (LIMA and RIMA) and/or venous grafts were used as graft material. Distal anastomoses were connected during the total aortic cross clamp time. Proximal anastomoses were performed within reperfusion time using a partial clamping technique of the ascending aorta. Finally, after decannulation, insertion of chest drains and sternal closure intubated patients were transferred to the intensive care unit (ICU).

\section{Application of cardioplegia}

In terms of cardioplegia protocol in our institution, the so called "cold induction" and "cold reinfusion" Buckberg cardioplegia is normally used. The components are supplied separately in two bottles $(400 \mathrm{~mL}$ and $100 \mathrm{~mL})$ and are first mixed together when needed. The induction cardioplegia solution contains: $400 \mathrm{~mL}$ of high potassium concentration with $\mathrm{KCl} 34 \mathrm{mmol}$, THAM $17.45 \mathrm{mmol}$ and $\mathrm{NaCl} 16.12 \mathrm{mmol}$; following substances are added with the second $100 \mathrm{~mL}$ bottle: citric acid $0.45 \mathrm{mmol} \times \mathrm{H}_{2} \mathrm{O}$, sodium citrate $2.60 \mathrm{mmol} \times 2 \mathrm{H}_{2} \mathrm{O}$, sodium hydrogen phosphate $0.47 \mathrm{mmol} \times 2 \mathrm{H}_{2} \mathrm{O}$ and glucose $92.42 \mathrm{mmol} \mathrm{x} \mathrm{H}_{2} \mathrm{O}$. For the reinfusion cardioplegia there are also two bottles that are first mixed when needed: $400 \mathrm{~mL}$ low potassium concentration of $\mathrm{KCl}$ $16.90 \mathrm{mmol}$, THAM $18.30 \mathrm{mmol}$ and $\mathrm{NaCl} 16.90 \mathrm{mmol}$. As with induction cardioplegia, additional substances are 
added from the $100 \mathrm{~mL}$ bottle: citric acid $0.48 \mathrm{mmol}$, sodium citrate $2.73 \mathrm{mmol}$, sodium hydrogen phosphate $0.49 \mathrm{mmol}$ and glucose $96.93 \mathrm{mmol}$. This concentrated solution is diluted in a ratio 1:4 with blood to provide oxygen, the desired $\mathrm{pH}$ and osmolarity. The cardioplegic induction solution with high potassium concentration is delivered over $4 \mathrm{~min}$ to initiate cardiac arrest whereas the following reinfusion solution with lower potassium concentration is delivered over 2 min to maintain cardiac arrest every $15-20 \mathrm{~min}$.

Cardioplegia was delivered into the aortic root immediately after cross clamping ascending aorta and reinfused into vein grafts after completion of the distal anastomosis in patients with severely obstructed or occluded coronary arteries. In the ICC group, cardiac arrest was initiated by cold induction using a mixture of the patient's own blood with the crystalloid Buckberg solution (Dr. Franz Köhler Chemie GmbH, Germany) at a ratio of 1 to 4 and blood flow rate of $200-300 \mathrm{~mL} / \mathrm{min}$. Patients were receiving cold $\left(4-6{ }^{\circ} \mathrm{C}\right)$ blood cardioplegia for $4 \mathrm{~min}$ aiming for a potassium target dose of $20 \mathrm{mmol} / \mathrm{L}$. In the ICC group the body temperature was routinely cooled down to $34{ }^{\circ} \mathrm{C}$. According to our institutional policy there was no additional topical cooling. While the aorta was cross clamped, intermittent reinfusions for 2-4 min each with ICC were applied at intervals of 15-20 min. Despite recommendations by the Buckberg protocol warm terminal reperfusion ('hot shot') was not infused prior to aortic unclamping in our center. Over the study period a total of 8 surgeons were performing elective and emergent CABG. Whereas 3 surgeons were advocates of Buckberg cardioplegia (ICC), other 5 surgeons were proponents of Calafiore cardioplegia (IWC). There were no surgeons who had used both types of cardioplegia dependent on clinical factors. Also, as it is a retrospective analysis, the choice of cardioplegia could not be influenced.

According to our institutional policy the body temperature using IWC was maintained between $36{ }^{\circ} \mathrm{C}$ and $37{ }^{\circ} \mathrm{C}$. In the IWC group, infusion of normothermic $\left(37^{\circ} \mathrm{C}\right)$ oxygenated blood was administered to the aortic root using a standardized setup. A syringe pump was connected to the extracorporeal circulation containing a modified mixture of $30 \mathrm{~mL}$ of $\mathrm{KCl}(2 \mathrm{mmol} / \mathrm{L})$ and $10 \mathrm{ml}$ of $\mathrm{MgSO} 4(2 \mathrm{mmol} / \mathrm{L})$. Cardiac arrest was induced by a syringe pump with the flow rate of $150 \mathrm{~mL} / \mathrm{h}$ at a blood flow rate of $200-300 \mathrm{~mL} / \mathrm{min}$ for approximately $2 \mathrm{~min}$ with the aim to achieve potassium concentration of $20 \mathrm{mmol} / \mathrm{L}$ and magnesium target dose of $5 \mathrm{mmol} / \mathrm{L}$. In cases when cardiac arrest was not achieved immediately, an additional bolus injection of 3-4 $\mathrm{ml}$ cardioplegia was infused. Thereafter, the cardioplegic solution was repeatedly administered every $15 \mathrm{~min}$ or after completion of each distal anastomosis, whereby the flow rate of the syringe pumps was reduced (120-90-60 mL/h) according the protocol of Calafiore et al. [5].

\section{Data collection}

All data were collected from our institutional database. The Electronic Patient Record of our department contains all patient data, such as demographics, preoperative risk factors, intra- and postoperative data. Moreover, the preoperative medication, especially the use of aspirin and clopidogrel and their discontinuation were recorded. In addition, laboratory values were obtained from the institutional database.

\section{Definitions}

Thirty day mortality was defined as death of any cause within 30 days after CABG. Perioperative myocardial infarction (PMI) was defined as the combination of the two following criteria proposed by Liakopoulos et al. [8] higher than fivefold increase in creatine kinase MB (CK-MB $>120$ $\mathrm{U} / \mathrm{L}$ ) including CK-MB fraction range of $6-25 \%$ and an increase in Troponin $\mathrm{T}$ over $1.5 \mathrm{ng} / \mathrm{mL}$ within $72 \mathrm{~h}$ after surgery. Further studies have also shown that those laboratory tests are qualified for the diagnostics of PMI in patients undergoing cardiac surgery, especially Troponin $\mathrm{T}$ [10-12].

\section{Statistical analysis}

Statistical analysis was performed using the IBM SPSS statistics version 19 (SPSS Inc., Chicago, IL, USA). Continuous variables were expressed as mean \pm standard deviation and categorical variables given as absolute values and percentages. A comparison of the two groups was done using ANOVA for continuous variables and Chi square test or Fisher exact test for categorical variables. $P$ values of $<0.05$ were considered statistically significant. Univariate analysis was executed to demonstrate associations between outcome and perioperative variables. Binary logistic regression analysis was performed to detect independent predictors for 30-day mortality. Kaplan-Meier Survival analysis was performed with the view to presenting long-term outcomes of the entire patient cohort as wall as comparing outcomes in subgroups.

\section{Results}

Patient demographics and preoperative baseline characteristics are listed in Table 1 . There were no statistically significant differences between ICC and IWC groups except for proportion of patients on regular statin medication $(66.7 \%$ in the ICC group vs. $80.7 \%$ in the IWC group, $p=0.035$ ) in the emergent subgroup and with the presence of left main disease with more than $50 \%$ stenosis $(34.5 \%$ in the ICC group vs. $23.5 \%$ in the IWC group, $p=0.026)$ in the elective subgroup. Patients were 
Table 1 Preoperative data, emergent vs. elective CABG devided in ICC and IWC

\begin{tabular}{|c|c|c|c|c|c|c|c|c|c|c|}
\hline & \multicolumn{5}{|c|}{ Emergent $\mathrm{CABG}$} & \multicolumn{5}{|c|}{ Elective CABG } \\
\hline & \multicolumn{2}{|l|}{$\overline{I C C}$} & \multicolumn{2}{|l|}{ IWC } & \multirow[b]{2}{*}{$\mathrm{p}$-Wert } & \multicolumn{2}{|l|}{$\overline{I C C}$} & \multicolumn{2}{|l|}{ IWC } & \multirow[b]{2}{*}{ p-Wert } \\
\hline & $\bar{n}$ & $\%$ & $\mathrm{n}$ & $\%$ & & $\mathrm{n}$ & $\%$ & $\mathrm{n}$ & $\%$ & \\
\hline Age $>70$ & 23 & 36.5 & 63 & 36.2 & & 45 & 39.8 & 117 & 35.2 & \\
\hline Female gender & 14 & 22.2 & 36 & 20.7 & 0.857 & 24 & 21.2 & 71 & 21.4 & 1.000 \\
\hline HT & 49 & 27.8 & 142 & 28.2 & 0.450 & 105 & 59.7 & 295 & 58.3 & 0.279 \\
\hline Pulm. HT & 6 & 9.5 & 11 & 6.5 & 0.410 & 4 & 3.5 & 12 & 3.6 & 1.000 \\
\hline Hyperlipidemia & 46 & 73.0 & 12 & 65.1 & 0.276 & 92 & 81.4 & 261 & 78.6 & 0.592 \\
\hline Diab. mellitus & 16 & 25.4 & 58 & 33.5 & 0.269 & 38 & 33.6 & 134 & 40.4 & 0.220 \\
\hline $\mathrm{BMI}>30$ & 14 & 22.2 & 43 & 24.7 & 0.734 & 42 & 37.2 & 98 & 29.5 & 0.159 \\
\hline $\mathrm{AF}$ & 14 & 23.7 & 33 & 22.2 & 0.857 & 5 & 4.4 & 20 & 6.0 & 0.641 \\
\hline $3 \mathrm{~V}-\mathrm{CAD}$ & 50 & 79.4 & 136 & 78.2 & 1.000 & 97 & 85.8 & 274 & 82.5 & 0.467 \\
\hline LMS & 31 & 49.2 & 81 & 47.1 & 0.883 & 39 & 34.5 & 78 & 23.5 & 0.026 \\
\hline COPD & 4 & 6.3 & 20 & 11.6 & 0.332 & 15 & 13.3 & 35 & 10.5 & 0.490 \\
\hline PAD & 10 & 15.9 & 31 & 17.9 & 0.847 & 20 & 17.7 & 52 & 15.7 & 0.658 \\
\hline Smoker & 30 & 47.6 & 91 & 52.9 & 0.556 & 46 & 40.7 & 137 & 41.3 & 1.000 \\
\hline Crea $>2$ & 5 & 7.9 & 11 & 6.3 & 0.770 & 2 & 1.8 & 11 & 3.3 & 0.317 \\
\hline CVA & 8 & 12.7 & 31 & 18.0 & 0.429 & 12 & 13.3 & 57 & 17.2 & 0.377 \\
\hline Beta-blocker & 48 & 76.2 & 139 & 81.3 & 0.462 & 86 & 76.1 & 274 & 82.5 & 0.165 \\
\hline Statin & 42 & 66.7 & 138 & 80.7 & 0.035 & 92 & 81.4 & 256 & 77.1 & 0.359 \\
\hline Antiplatel. & 55 & 91.7 & 156 & 94.5 & 0.532 & 26 & 23.0 & 54 & 16.3 & 0.120 \\
\hline$E F<40 \%$ & 23 & 36.5 & 48 & 27.6 & & 33 & 29.2 & 97 & 29.2 & \\
\hline Euroscore Logistic & \multicolumn{2}{|c|}{$29.82 \pm 27.14$} & \multicolumn{2}{|c|}{$25.09 \pm 23.95$} & 0.214 & \multicolumn{2}{|c|}{$7.26 \pm 10.91$} & \multicolumn{2}{|c|}{$7.20 \pm 9.48$} & 0.953 \\
\hline
\end{tabular}

comparable in terms of past medical history, cardiac risk factors, preoperative medication and logistic Euroscore.

Table 2 represents intra-operative patient data for both groups. In the emergent subgroup, patients receiving IWC had longer cross clamp time $(41.1 \pm 13.9$ vs. $35.4 \pm 12.2 \mathrm{~min}, p=0.005$ ), whereas patients receiving ICC were associated with significantly longer administration time $(5.9 \pm 2,2$ vs. $5.0 \pm 2.7 \mathrm{~min}, p=0.017)$ and amount ( $329 \pm 155$ vs. $18.5 \pm 5.6 \mathrm{ml}, p<0.001)$ of cardioplegia compared to IWC group. Similar associations were also found within the elective subgroup. In terms of intraoperative data within both subgroups there were no statistically significant differences in CPB, operation and, reperfusion time, and the usage of IMA. Groups were also comparable regarding phosphodiesterase-III-inhibitor usage, and the need for hemofiltration.

Postoperative patients' characteristics are presented in Table 3. The mean number of platelet units transfused in the elective subgroup was $0.62 \pm 1.43$ in the ICC group and $0.31 \pm 0.72$ in the IWC group $(p=0.003)$. The 30-day all cause mortality in the elective subgroup tended to be increased in the IWC group with $2 \%$ in contrast to $0 \%$ in ICC group, however it did not reach statistical significance $(p=0.072)$. Similarly, in the emergent subgroup, the 30 -day mortality was $5,1 \%$ in the ICC group vs. $4,2 \%$ in the IWC group not reaching statistical significance. During cardiac surgery in the emergent subgroup $10.8 \%$ patients of the ICC group received an intra-aortic balloon pump (IABP) compared to $6.5 \%$ of the IWC group also not reaching statistical significance $(p=0.077)$. Further outcome variables, such as PMI, length of hospital and ICU stay, ventilation duration, extracorporeal membrane oxygenation (ECMO), dialysis, inotropic support, blood transfusion and total blood loss were also not statistically different. Long-term outcomes in terms of overall cumulative survival are presented in Figure 1. The overall 30-day, 1-, 3- and 6-year survival of the entire patient cohort was 93.7, 91.8, 90.4 and $89.1 \%$, respectively, with significantly better outcomes when operated electively $(p<0.001)$ but no differences between ICC and IWC both in elective $(p=0.857)$ and emergent $(p=0.741)$ subgroups.

Univariate regression analysis was executed (Tables 4 and 5), in order to show possible associations of early 
Table 2 Intraoperative data, emergent vs. elective CABG devided in ICC and IWC

\begin{tabular}{|c|c|c|c|c|c|c|c|c|c|c|}
\hline & \multicolumn{5}{|c|}{ Emergent CABG } & \multicolumn{5}{|c|}{ Elective CABG } \\
\hline & \multicolumn{2}{|c|}{ ICC } & \multicolumn{2}{|l|}{ IWC } & \multirow[b]{2}{*}{$p$-Wert } & \multicolumn{2}{|l|}{ ICC } & \multicolumn{2}{|l|}{ IWC } & \multirow[b]{2}{*}{ p-Wert } \\
\hline & $\mathrm{n}$ & $\%$ & $\mathrm{n}$ & $\%$ & & $\mathrm{n}$ & $\%$ & $\mathrm{n}$ & $\%$ & \\
\hline $\mathrm{AOX}$ (min) & \multicolumn{2}{|c|}{$35.38 \pm 12.16$} & \multicolumn{2}{|c|}{$41.05 \pm 13.91$} & 0.005 & \multicolumn{2}{|c|}{$39.92 \pm 12.65$} & \multicolumn{2}{|c|}{$43.96 \pm 15.85$} & 0.015 \\
\hline CBP (min) & \multicolumn{2}{|c|}{$81.81 \pm 37.75$} & \multicolumn{2}{|c|}{$90.50 \pm 38.68$} & 0.121 & \multicolumn{2}{|c|}{$77.96 \pm 25.20$} & \multicolumn{2}{|c|}{$82.93 \pm 32.25$} & 0.138 \\
\hline OP-time (min) & \multicolumn{2}{|c|}{$182.03 \pm 46.10$} & \multicolumn{2}{|c|}{$198.52 \pm 64.67$} & 0.064 & \multicolumn{2}{|c|}{$184.39 \pm 46.24$} & \multicolumn{2}{|c|}{$201.31 \pm 95.14$} & 0.092 \\
\hline Number of distal grafts & \multicolumn{2}{|c|}{$2.94 \pm 0.76$} & \multicolumn{2}{|c|}{$3.09 \pm 0.91$} & 0.245 & \multicolumn{2}{|c|}{$3.01 \pm 0.84$} & \multicolumn{2}{|c|}{$2.93 \pm 0.78$} & 0.386 \\
\hline LIMA use & 58 & 92.1 & 158 & 90 & 1.000 & 107 & 94.7 & 315 & 94.9 & 1.000 \\
\hline RIMA use & 7 & 11.1 & 27 & 15.5 & 0.530 & 28 & 24.8 & 83 & 25.0 & 1.000 \\
\hline Reperfusion time (min) & \multicolumn{2}{|c|}{$34.87 \pm 22.05$} & \multicolumn{2}{|c|}{$38.35 \pm 27.35$} & 0.366 & \multicolumn{2}{|c|}{$28.54 \pm 12.69$} & \multicolumn{2}{|c|}{$30.72 \pm 17.55$} & 0.225 \\
\hline Cardioplegia time (min) & \multicolumn{2}{|c|}{$5.91 \pm 2.22$} & \multicolumn{2}{|c|}{$5.01 \pm 2.68$} & 0.017 & \multicolumn{2}{|c|}{$7.03 \pm 3.05$} & \multicolumn{2}{|c|}{$5.08 \pm 2.73$} & 0.000 \\
\hline Cardioplegia in (mL) & \multicolumn{2}{|c|}{$329.02 \pm 155.43$} & \multicolumn{2}{|c|}{$18.54 \pm 5.6$} & 0.000 & \multicolumn{2}{|c|}{$370 \pm 125.60$} & \multicolumn{2}{|c|}{$19.15 \pm 21.51$} & 0.000 \\
\hline Hemofiltration & 22 & 34.9 & 48 & 27.6 & 0.333 & 13 & 11.5 & 37 & 11.1 & 1.000 \\
\hline PDI (Corotrop) & 21 & 34.4 & 63 & 36.4 & 0.877 & 13 & 15.9 & 75 & 22.6 & 0.143 \\
\hline
\end{tabular}

Intraoperative patients data: emergent- vs. elective CABG devided in intermittent cold cardioplegia (ICC) and intermittent warm cardioplegia (IWC). Aortic clamp time (AOX), cardiopumonary bybass (CBP), LIMA (left internal mammary artery), RIMA (right internal mammary artery), phospodiesterase inhibitor (PDI)

clinical outcome with determined variables. There were no associations found between each clinical endpoint and the application of IWC or ICC.

In order to detect independent preoperative predictors for 30-day mortality and PMI binary logistic regression analysis was performed (Table 6 and 7) using only preoperative variables. Multivariate analysis revealed that $\mathrm{EF}<40 \%$ was the strongest independent predictor for 30-day mortality (OR 3.66; 95 \%-CI 1.79-7.52; $p<0.001$ ), followed by atrial fibrillation, PAD and anti-platelet drugs not suspended at least 5 days before surgery (Table 6). In contrast, three independent predictor

Table 3 Postoperative data, emergent vs. elective CABG devided in ICC and IWC

\begin{tabular}{|c|c|c|c|c|c|c|c|c|c|c|}
\hline & \multicolumn{5}{|c|}{ Emergent $\mathrm{CABG}$} & \multicolumn{5}{|c|}{ Elective CABG } \\
\hline & \multicolumn{2}{|c|}{ ICC } & \multicolumn{2}{|c|}{ IWC } & \multirow[b]{2}{*}{$p$-value } & \multicolumn{2}{|c|}{ ICC } & \multicolumn{2}{|c|}{ IWC } & \multirow[b]{2}{*}{$p$-value } \\
\hline & $n$ & $\%$ & $n$ & $\%$ & & $n$ & $\%$ & $n$ & $\%$ & \\
\hline 30 day mortality & 9 & 5.1 & 31 & 4.7 & 0.661 & 0 & 0.0 & 10 & 2.0 & 0.072 \\
\hline PMl & 12 & 6.8 & 28 & $U$ & 0.563 & 13 & 7.4 & 31 & 6.1 & 0.584 \\
\hline ICU(d) & \multicolumn{2}{|c|}{$6.44 \pm 11.80$} & \multicolumn{2}{|c|}{$5.73 \pm 5.70$} & 0.536 & \multicolumn{2}{|c|}{$3.83 \pm 4.15$} & \multicolumn{2}{|c|}{$4.01 \pm 4.50$} & 0.708 \\
\hline Hospital (d) & \multicolumn{2}{|c|}{$13.19 \pm 12.77$} & \multicolumn{2}{|c|}{$13.53 \pm 9.39$} & 0.825 & \multicolumn{2}{|c|}{$12.98 \pm 7.21$} & \multicolumn{2}{|c|}{$13.05 \pm 6.20$} & 0.979 \\
\hline Duration of ventilation (h) & \multicolumn{2}{|c|}{$109.85 \pm 171.87$} & \multicolumn{2}{|c|}{$76.85 \pm 150.32$} & 0.194 & \multicolumn{2}{|c|}{$44.45 \pm 168.25$} & \multicolumn{2}{|c|}{$30.97 \pm 73.03$} & $0.24 /$ \\
\hline \multicolumn{11}{|l|}{ IABP } \\
\hline - preop & 10 & 5.7 & 19 & 3.8 & 0.370 & 1 & 0.6 & 2 & 0.4 & 1.000 \\
\hline - intraop & 19 & 10.8 & 33 & 6.5 & 0.077 & 2 & 1.1 & 8 & 1.6 & 1.000 \\
\hline - postop & 3 & 1.7 & 9 & 1.8 & 1.000 & 1 & 0.6 & 5 & 1.0 & 1.000 \\
\hline ECMO & 5 & 2.8 & 11 & 2.2 & 0.770 & 0 & 0.0 & 0 & 0.0 & \\
\hline \multicolumn{11}{|l|}{ Blood transfusion } \\
\hline$-\mathrm{RBC}$ & \multicolumn{2}{|c|}{$7.76 \pm 12.92$} & \multicolumn{2}{|c|}{$5.96 \pm 7.30$} & 0.183 & \multicolumn{2}{|c|}{$3.93 \pm 5.79$} & \multicolumn{2}{|c|}{$3.45 \pm 5.07$} & 0.401 \\
\hline - FFP & \multicolumn{2}{|c|}{$4.43 \pm 6.8$} & \multicolumn{2}{|c|}{$2.79 \pm 4.21$} & 0.210 & \multicolumn{2}{|c|}{$1.50 \pm 3.95$} & \multicolumn{2}{|c|}{$1.19 \pm 3.29$} & 0.411 \\
\hline - Platelets & \multicolumn{2}{|c|}{$1.46 \pm 1.45$} & \multicolumn{2}{|c|}{$1.05 \pm 1.10$} & 0.220 & \multicolumn{2}{|c|}{$0.62 \pm 1.43$} & 0.31 & & 0.003 \\
\hline Total blood loss (ml) & 153 & 049.81 & 140 & 019.14 & 0.425 & 108 & 715.66 & 962. & 22.91 & 0.084 \\
\hline Dialysis & 3 & 1.7 & 21 & 4.2 & 0.142 & 3 & 1.7 & 11 & 2.2 & 1.000 \\
\hline Inotropie support > 24 h & 14 & 8.9 & 48 & 10.1 & 0.858 & 23 & 14.6 & 56 & 11.7 & 0.390 \\
\hline Inotropie support > 48 h & 17 & 10.8 & 58 & 12.2 & 0.734 & 12 & 7.6 & 52 & 10.9 & 0.274 \\
\hline
\end{tabular}




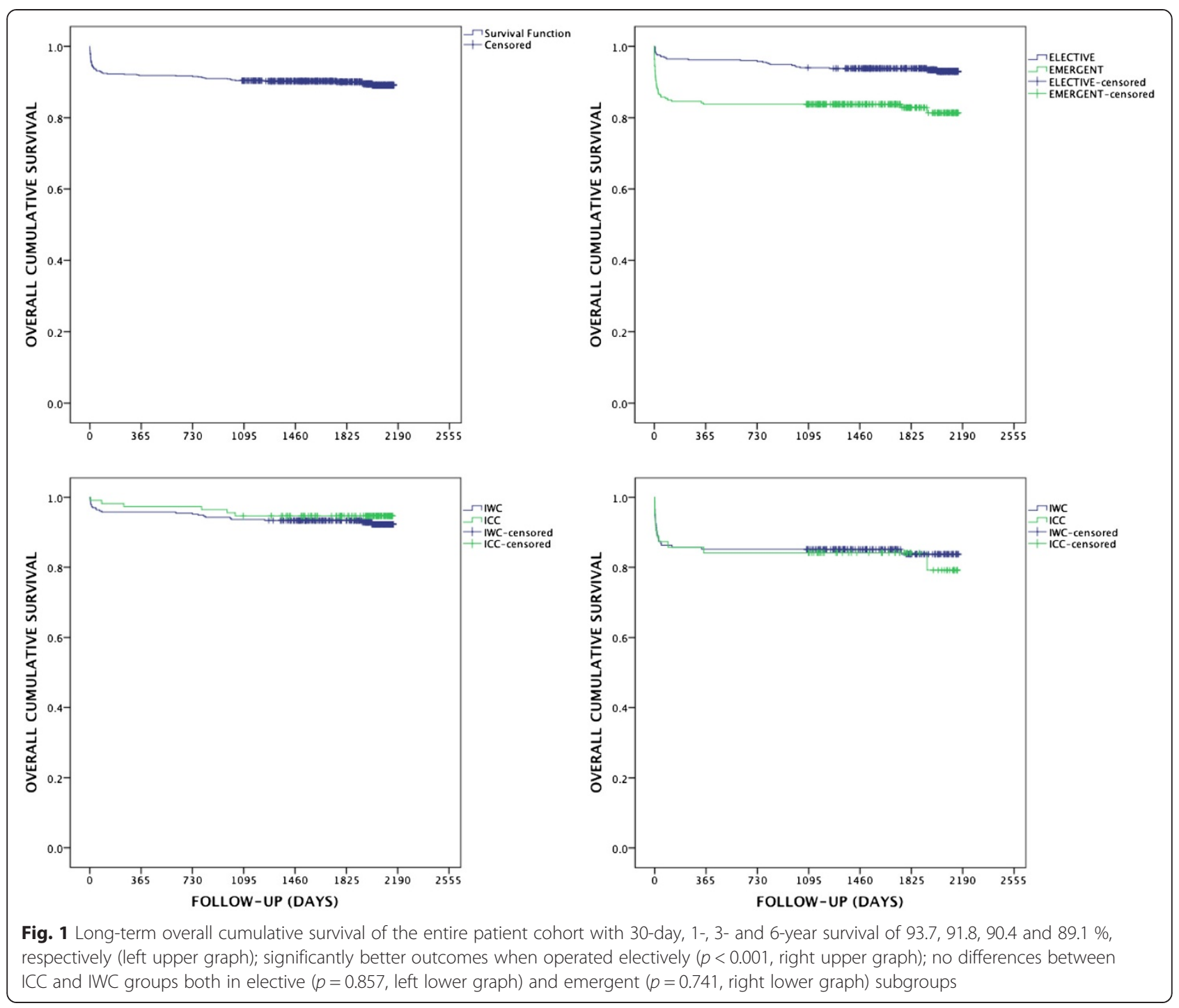

variables showed a protective influence on 30-day mortality, namely 3-vessel-disease (OR 0.36; 95 \%-CI 0.16-083; $p=0.016$ ), preoperative statin therapy and dyslipidemia. The strongest independent predictor for PMI appeared to be preoperative anti-platelet drug therapy (OR 4.79; $95 \%$-CI 3.10-7.40; $p<0.001$ ), followed by IABP, hemofiltration, $\mathrm{EF}<40 \%$, atrial fibrillation and cerebrovascular disease (Table 7). Preoperative statin therapy tended to be a borderline predictor with a protective influence on PMI (OR 0.66; 95 \%-CI 0.41-1.08; $p=0.101$ ).

\section{Analysis of cause of death}

In the IWC and ICC emergent CABG group 8 patients vs. 5 patients died after acute myocardial infarction with postcardiotomy ECMO, 2 of them had cerebrovascular events, 4 patients vs. 1 patient died due to severe sepsis with multiorgan failiure, 2 patients vs 0 patients with mesenterial ischemia and laparotomy, 1 patients vs. 0 patients with LV and LA thrombus in association with heparin induced thrombocytopenia and 6 patients vs. 3 patients died postoperatively after CPR due to electromechanical dissociation and low-output syndrome, respectively.

On the other hand, in the IWC and ICC elective CABG group 3 patients vs. 0 patients died after pericardial tamponade or pulmonary emboli with postcardiotomy ECMO, 3 patients vs. 0 patients died due to severe sepsis and multiorgan failiure, 1 patient vs 0 patients after mesenterial ischemia and laparotomy in association with sepsis and 3 patients vs. 0 patients postoperatively after CPR due to electromechanical dissociation and low-output syndrome, respectively. 
Table 4 Univariate analysis, primary endpoint: 30-days mortality

\begin{tabular}{|c|c|c|c|c|c|c|c|}
\hline \multicolumn{8}{|c|}{ Univariate analysis, primary endpoint: 30-days mortality $(n=45)$} \\
\hline \multirow[t]{2}{*}{ Variable } & \multicolumn{2}{|c|}{ Yes } & \multicolumn{2}{|l|}{ No } & \multirow[t]{2}{*}{ O.R. } & \multirow[t]{2}{*}{$95 \%$-C.I. } & \multirow[t]{2}{*}{$p$-value } \\
\hline & $n$ & $\%$ & $n$ & $\%$ & & & \\
\hline Age $>70$ y & 17 & 37.8 & 28 & 6.4 & 1.04 & $(0.56-1.95)$ & 0.875 \\
\hline Female & 13 & 28.9 & 32 & 5.9 & 1.55 & $(0.79-3.03)$ & 0.192 \\
\hline HT & 35 & 79.5 & 9 & 9.9 & 0.55 & $(0.26-1.22)$ & 0.163 \\
\hline pulmonal. HT & 7 & 16.3 & 36 & 5.5 & 4.44 & $(1.81-10.89)$ & 0.003 \\
\hline HLP & 21 & 47.7 & 23 & 13.4 & 0.27 & $(0.15-0.51)$ & $<0,001$ \\
\hline Diab. mellitus & 18 & 40.0 & 27 & 6.1 & 1.18 & $(0.64-2.19)$ & 0.632 \\
\hline $\mathrm{BMI}>30$ & 13 & 28.9 & 32 & 6.5 & 1.01 & $(0.52-1.96)$ & 1.000 \\
\hline $\mathrm{AF}$ & 15 & 34.1 & 29 & 4.8 & 4.60 & $(2.34-9.03)$ & $<0,001$ \\
\hline $3 \mathrm{~V}-\mathrm{CAD}$ & 32 & 71.1 & 13 & 10.1 & 0.54 & $(0.27-1.05)$ & 0.074 \\
\hline LMS & 20 & 44.4 & 25 & 5.4 & 1.63 & $(0.89-3.00)$ & 0.141 \\
\hline COPD & 9 & 20.0 & 36 & 5.8 & 2.13 & $(0.99-4.62)$ & 0.080 \\
\hline PAD & 15 & 33.3 & 30 & 5.2 & 2.62 & $(1.36-5.03)$ & 0.007 \\
\hline Smoker & 22 & 50.0 & 22 & 5.7 & 1.27 & $(0.59-2.33)$ & 0.531 \\
\hline Crea $>2$ & 6 & 14.0 & 37 & 5.7 & 4.32 & $(1.66-11.24)$ & 0.007 \\
\hline Renal insuff. preop. Crea $<2$ & 23 & 51.1 & 22 & 4.7 & 2.25 & $(1.23-4.14)$ & 0.013 \\
\hline CVA & 6 & 13.6 & 38 & 6.6 & 0.79 & $(0.33-1.91)$ & 0.333 \\
\hline Beta-blocker & 27 & 62.8 & 16 & 11.8 & 0.38 & $(0.20-0.73)$ & 0.005 \\
\hline Statin & 22 & 51.2 & 21 & 13.6 & 0.27 & $(0.14-0.51)$ & $<0,001$ \\
\hline Antiplatelet & 29 & 67.4 & 14 & 3.6 & 2.90 & $(1.50-5.59)$ & $<0,001$ \\
\hline$E F<40 \%$ & 30 & 66.7 & 15 & 3.1 & 5.36 & $(2.82-10.21)$ & $<0,001$ \\
\hline EF $40-50 \%$ & 8 & 17.8 & 37 & 7.0 & 0.70 & $(0.32-1.53)$ & 0.466 \\
\hline$E F>50 \%$ & 7 & 15.6 & 38 & 10.4 & 0.19 & $(0.08-0.43)$ & $<0,001$ \\
\hline Euroscore $>=6$ & 40 & 95.2 & 2 & 0.6 & 16.98 & $(4.07-70.85)$ & $<0,001$ \\
\hline IABP preop. & 6 & 13.3 & 39 & 5.9 & 3.68 & $(1.43-9.45)$ & 0.013 \\
\hline NYHA III + IV & 34 & 75.6 & 11 & 3.3 & 2.96 & $(1.48-5.94)$ & 0.002 \\
\hline Emergent $C A B G$ & 34 & 75.6 & 11 & 2.5 & 6.43 & $(3.15-12.76)$ & $<0,001$ \\
\hline preop. myocardial infarction & 22 & 50.0 & 22 & 4.4 & 3.26 & $(1.76-6.06)$ & $<0,001$ \\
\hline \multicolumn{8}{|c|}{ Time between angiography and operation: } \\
\hline$<3 d$ & 27 & 65.9 & 14 & 3.2 & 4.41 & $(2.26-8.60)$ & $<0,001$ \\
\hline$<24 \mathrm{~h}$ & 26 & 63.4 & 15 & 3.1 & 5.79 & $(2.99-11.24)$ & $<0,001$ \\
\hline OP-time after 08.00 pm & 6 & 13.3 & 39 & 6.2 & 1.57 & $(0.64-3.86)$ & 0.292 \\
\hline ICC cardioplegia & 10 & 23.8 & 32 & 6.3 & 0.89 & $(0.43-1.86)$ & 0.857 \\
\hline IWC cardioplegia & 31 & 73.8 & 11 & 6.2 & 0.99 & $(0.48-2.00)$ & 1.000 \\
\hline AoX $<80 \mathrm{~min}$ & 37 & 88.1 & 5 & 35.7 & 0.11 & $(0.030 .33)$ & 0.001 \\
\hline AoX $>60 \mathrm{~min}$ & 6 & 14.3 & 36 & 5.9 & 1.39 & $(0.57-3.43)$ & 0.444 \\
\hline AoX $>45$ min & 13 & 31.0 & 29 & 6.3 & 0.91 & $(0.46-1.78)$ & 0.866 \\
\hline CPB $>180 \mathrm{~min}$ & 10 & 22.7 & 34 & 5.0 & 37.82 & (12.25 116.80) & $<0,001$ \\
\hline $\mathrm{CPB}>120 \mathrm{~min}$ & 20 & 45.5 & 24 & 3.9 & 9.36 & $(4.85-18.04)$ & $<0,001$ \\
\hline OP-time $>180 \mathrm{~min}$ & 33 & 73.3 & 12 & 3.9 & 2.47 & $(1.25-4.88)$ & 0.008 \\
\hline Reperfusion time $>60 \mathrm{~min}$ & 17 & 39.5 & 26 & 4.1 & 13.40 & $(6.57-27.34)$ & $<0,001$ \\
\hline Hemofiltration & 27 & 60.0 & IS & 3.2 & 8.54 & $(4.53-16.10)$ & $<0,001$ \\
\hline PDI (Corotrop) & 24 & 54.5 & 20 & 2.9 & 3.71 & $(2.00-6.90)$ & $<0,001$ \\
\hline
\end{tabular}


Table 4 Univariate analysis, primary endpoint: 30-days mortality (Continued)

\begin{tabular}{|c|c|c|c|c|c|c|c|}
\hline PMI & 36 & 80.0 & 9 & 1.7 & 14.71 & $(6.92-31.26)$ & $<0,001$ \\
\hline$I C U>3 d$ & 29 & 69.0 & 13 & 3.3 & 3.28 & $(1.67-6.43)$ & $<0,001$ \\
\hline$I C U>5 d$ & 19 & 45.2 & 23 & 4.6 & 2.52 & $(8.83-34.82)$ & 0.006 \\
\hline $\mathrm{ICU}>7 \mathrm{~d}$ & 16 & 38.1 & 26 & 4.8 & 2.59 & $(1.35-4.97)$ & 0.005 \\
\hline$I C U>10 d$ & 12 & 28.6 & 30 & 5.1 & 3.13 & $(1.53-6.38)$ & 0.003 \\
\hline Hospital stay $>15 \mathrm{~d}$ & 10 & 22.7 & 34 & 6.3 & 1.07 & $(0.51-2.21)$ & 0.851 \\
\hline Ventilation time $>48 \mathrm{~h}$ & 23 & 62.2 & 14 & 2.6 & 11.23 & $(5.54-22.73)$ & $<0,001$ \\
\hline IABP preop. & 27 & 60.0 & 18 & 2.9 & 15.91 & $(8.25-30.67)$ & $<0,001$ \\
\hline ECMO & 15 & 33.3 & 30 & 4.4 & 107.83 & $(29.61-392.72)$ & $<0,001$ \\
\hline Blood transfusion & 43 & 100.0 & 0 & 0.0 & 0.92 & $(0.90-0.94)$ & 0.068 \\
\hline RBC Unit $>5$ & 31 & 68.9 & 14 & 2.7 & 8.48 & $(4.39-16.39)$ & $<0,001$ \\
\hline Total blood loss >1l & 19 & 65.6 & 10 & 2.7 & 2.62 & $(1.20-5.73)$ & 0.020 \\
\hline Total blood loss >2l & 12 & 41.4 & 17 & 3.0 & 6.17 & $(2.82-13.52)$ & $<0,001$ \\
\hline Dialysis & 12 & 26.7 & 33 & 5.0 & 8.08 & $(3.77-12.30)$ & $<0,001$ \\
\hline Inotropie support $>48$ h & 26 & 89.7 & 3 & 0.8 & 12.19 & (3.65 40.70) & $<0,001$ \\
\hline $\mathrm{ACT}>150 \mathrm{sec}$ & 10 & 41.7 & 14 & 3.1 & 4.33 & $(1.85-10.10)$ & $<0,001$ \\
\hline Pneumonia & 10 & 22.7 & 34 & 5.1 & 12.39 & $(5.19-29.62)$ & $<0,001$ \\
\hline Wound infection & 3 & 6.8 & 41 & 6.5 & 0.77 & $(0.23-2.57)$ & 1.000 \\
\hline Operative re-exploration & 13 & 29.5 & 31 & 4.8 & 6.91 & $(3.34-14.31)$ & $<0,001$ \\
\hline
\end{tabular}

Pre- intra- and postoperative data in univariate analysis. The nominal variables are shown in absolute and percentage figures. Cerebrovascular accident (CVA), 3 vessel coronary artery disease (3-V CAD), left main stenosis (LMS), Red blood cells (RBC)

\section{Discussion}

As several clinical studies have shown superior outcomes when using blood cardioplegia, this type of myocardial protection has been increasingly implemented in most centers including our department $[6,13]$. So far, most prior research has been particularly focused on cold and warm blood cardioplegia in elective CABG patients [14, 15]. Also the differences between crystalloid and cold blood cardioplegia have been largely debated in previous research $[16,17]$. In order to fill the gap in terms of analysis of differences in cold and warm blood cardioplegia in emergent setting, we conducted our study with particular attention to emergent CABG patients analysing the impact of different intermittent blood cardioplegia application. Previous research has indicated that there were no significant differences between antegrade and retrograde application of blood cardioplegia in terms of mortality and myocardial infarction [14, 18]. Due to easier technical aspects it has become the standard of care to administer antegrade blood cardioplegia for CABG procedures in our institution. In this study we analyzed different cardioplegia strategies in emergent compared to elective CABG patients. The intraoperative patient data showed similar results referring to $\mathrm{CPB}$, operation, and reperfusion time, usage of IMA and the need for hemodialysis. However, it is interesting to note that even though the aortic cross clamp time was significantly longer in the IWC group there were no differences in PMI. In a recent study it was even suggested that cold blood cardioplegia seems to be associated with more harmful effects on the myocardium in CABG patients when compared to warm blood cardioplegia [19]. In contrast, another study by Liakopoulos et al. demonstrated that cold blood cardioplegia according to Buckberg provided superior myocardial protection in terms of 30-day mortality, cardiac death and PMI in patients requiring prolonged aortic cross clamp time during cardiac surgery [9]. However, it is very doubtful as to whether a meaningful comparison can be made between CABG and non-CABG surgery procedures precisely because severe coronary stenosis may impair cardioplegic delivery leading to a different delivery of each solution beyond the stenosis.

Another study with prospective randomized design which was published two decades ago showed strong evidence that warm blood cardioplegia was superior to cold blood cardioplegia in CABG patients in terms of PMI [20]. As it was a randomized study, there were several exclusion criteria, such as patients with renal failure, or emergent patients who were not able to sign informed consent. In order to present a real world experience, we did not intend to exclude any cases, analyzing consecutive patients including those requiring preoperative mechanical ventilation or patients with renal failure. Moreover, an emergent subgroup was created and matched with consecutive patients from the elective 
Table 5 Univariate analysis, primary endpoint: Perimyocardial Infarction (PMI)

\begin{tabular}{|c|c|c|c|c|c|c|c|}
\hline \multicolumn{8}{|c|}{ Univariate analysis, primary endpoint: PMI $(n=175)$} \\
\hline \multirow[t]{2}{*}{ Variable } & \multicolumn{2}{|l|}{ Yes } & \multicolumn{2}{|l|}{ No } & \multirow[t]{2}{*}{ O.R. } & \multirow[t]{2}{*}{$95 \%-C . I}$. & \multirow[t]{2}{*}{$p$-value } \\
\hline & $n$ & $\%$ & $n$ & $\%$ & & & \\
\hline Age $>70$ years & 66 & $(37.7)$ & 109 & (24.8) & 1.05 & $(0.74-1.50)$ & 0.786 \\
\hline Female & 36 & $(20.6)$ & 139 & $(25.4)$ & 0.94 & $(0.62-1.44)$ & 0.832 \\
\hline HT & 141 & $(\mathrm{~S} 1.0)$ & 33 & $(35.3)$ & 0.54 & $(0.34-0.85)$ & 0.013 \\
\hline pulmonal HT & 14 & $(8.1)$ & 158 & $(24-2)$ & 2.19 & $(1.08-4.44)$ & 0.040 \\
\hline HLP & 122 & $(70.1)$ & 52 & $(30.2)$ & 0.71 & $(0.48-1.04)$ & 0.085 \\
\hline Diab. mellitus & 63 & $(36.0)$ & 112 & $(25.4)$ & 0.98 & $(0.69-1.40)$ & 0.928 \\
\hline $\mathrm{BMI}>30$ & 50 & $(25.6)$ & 125 & (25.3) & 0.99 & $(0.68-1.44)$ & 1.000 \\
\hline AF & 39 & $(22.9)$ & 131 & (21.6) & 3.46 & $(2.14-5.58)$ & $<0,001$ \\
\hline $3 \mathrm{~V}-\mathrm{CAD}$ & 140 & (SO.O) & 35 & $(27.1)$ & 0.88 & $(0.57-1.36)$ & 0.575 \\
\hline LMS & 73 & $(42-5)$ & 100 & $(21.8)$ & 1.67 & $(1.17-2.38)$ & 0.005 \\
\hline COPD & 20 & $(\mathrm{H}-4)$ & 155 & $(25.2)$ & 1.04 & $(0.61-1.79)$ & 0.890 \\
\hline PAD & 39 & $(22.3)$ & 136 & $(23.7)$ & 1.57 & $(1.02-2.41)$ & 0.048 \\
\hline Smoker & 84 & $(48.3)$ & 90 & $(23.4)$ & 1.23 & $(0.87-1.73)$ & 0.253 \\
\hline Crea $>2$ & 14 & $(8-5)$ & 151 & $(23.3)$ & 3.08 & $(1.45-6.52)$ & 0.006 \\
\hline Renal insuff. preop Crea $<2$ & 78 & $(44.6)$ & 97 & $(20.8)$ & 1.97 & $(1.38-2.80)$ & $<0,001$ \\
\hline CVA & 36 & $(20.7)$ & 138 & $(23.9)$ & 1.47 & $(0.95-2.28)$ & 0.098 \\
\hline Beta-blocker & 127 & $(73.4)$ & 46 & (33.3) & 0.58 & $(0.39-0.87)$ & 0.011 \\
\hline Statin & 117 & $(57.6)$ & 55 & $(96.4)$ & 0.49 & $(0.33-0.72)$ & $<0,001$ \\
\hline Antiplatelet & 127 & $(75.1)$ & 42 & $(10.9)$ & 6.19 & $(4.17-9.19)$ & $<0,001$ \\
\hline$E F<40 \%$ & SO & $(46.0)$ & 94 & (19.3) & 2.66 & $(1.85-3.80)$ & $<0,001$ \\
\hline EF $40-50 \%$ & 51 & $(29.3)$ & 123 & $(23.1)$ & 1.54 & $(1.05-2.27)$ & 0.038 \\
\hline$E F>50 \%$ & 43 & $(24.7)$ & 131 & $(35.7)$ & 0.27 & $(0.19-0.40)$ & $<0,001$ \\
\hline Euroscore $>=6$ & 138 & (S2.6) & 29 & (9.9) & 5.13 & $(3.31-7.93)$ & $<0,001$ \\
\hline IABP preop & 25 & (144) & 149 & $(22.6)$ & 12.25 & $(5.20-28.88)$ & $<0,001$ \\
\hline NYHA $111+$ IV & 116 & $(55.3)$ & 59 & (17.9) & 2.12 & $(1.49-3.04)$ & $<0,001$ \\
\hline Emergent CABG & 125 & (71.4) & 50 & $(11.2)$ & 8.16 & $(5.55-11.99)$ & $<0,001$ \\
\hline \multicolumn{8}{|c|}{ Time between angiography and operation } \\
\hline$<3 d$ & 100 & $(65.8)$ & 52 & $(11.9)$ & 6.61 & $(4.45-9.82)$ & $<0,001$ \\
\hline$<24 \mathrm{~h}$ & 92 & $(50.5)$ & 52 & $(12.4)$ & 8.77 & $(5.83-13.19)$ & $<0,001$ \\
\hline OP-time after 08.00 pm & 35 & $(20.0)$ & 60 & $(22.2)$ & 4.22 & $(2.49-7.15)$ & $<0,001$ \\
\hline ICC & 47 & $(28.0)$ & 121 & $(23.9)$ & 1.16 & $(0.79-1.72)$ & 0.477 \\
\hline IWC & 120 & $(71.4)$ & 48 & $(27.1)$ & 0.84 & $(0.57-1.23)$ & 0.363 \\
\hline AoX $<80 \mathrm{~min}$ & 164 & $(95.5)$ & 5 & $(42.9)$ & 0.43 & $(0.151 .25)$ & 0.122 \\
\hline AoX $>60 \mathrm{~min}$ & 17 & $(10.0)$ & 153 & $(25.0)$ & 0.88 & $(0.50-1.56)$ & 0.777 \\
\hline AoX $>45 \mathrm{~min}$ & 53 & $(31.2)$ & 117 & $(25.4)$ & 0.90 & $(0.62-1.30)$ & 0.574 \\
\hline $\mathrm{CPB}>180 \mathrm{~min}$ & 13 & $(7.5)$ & 161 & $(23.8)$ & 20.83 & $(4.65-93.29)$ & $<0,001$ \\
\hline $\mathrm{CPB}>120 \mathrm{~min}$ & 42 & $(24.1)$ & 132 & $(21.3)$ & 5.00 & $(3.03-8.26)$ & $<0,001$ \\
\hline OP-time > $180 \mathrm{~min}$ & 111 & (54 2) & 62 & $(20.3)$ & 1.75 & $(1.23-2.51)$ & 0.002 \\
\hline Reperfusion time $>60 \mathrm{~min}$ & 35 & $(20.5)$ & 136 & $(21.2)$ & 10.83 & $(5.47-21.43)$ & $<0,001$ \\
\hline Hemofiltration & 59 & $(39.4)$ & 106 & $(18.6)$ & 5.49 & $(3.64-8.29)$ & $<0,001$ \\
\hline PDI (Corotrop) & 81 & $(53.2)$ & 92 & $(18.1)$ & 3.64 & $(2.51-5.26)$ & $<0,001$ \\
\hline 30-days mortiality & 36 & (20.6) & 139 & (21.4) & 14.71 & $(6.92-31.25)$ & $<0,001$ \\
\hline
\end{tabular}


Table 5 Univariate analysis, primary endpoint: Perimyocardial Infarction (PMI) (Continued)

\begin{tabular}{|c|c|c|c|c|c|c|c|}
\hline$I C U>3 d$ & 118 & (72.0) & 46 & $(11.8)$ & 5.27 & $(3.58-7.76)$ & $<0,001$ \\
\hline$I C U>5 d$ & 36 & $(52.4)$ & 78 & (15.6) & 5.18 & $(3.54-7.59)$ & $<0,001$ \\
\hline $\mathrm{ICU}>7 \mathrm{~d}$ & 72 & $(43.9)$ & 92 & $(17.1)$ & 5.30 & $(3.55-7.93)$ & $<0,001$ \\
\hline$I C U>10 d$ & 46 & (32.0) & 118 & (19.9) & 4.87 & $(3.03-7.83)$ & $<0,001$ \\
\hline Hospital stay $>15 d$ & 55 & (32.0) & 117 & (21.7) & 2.11 & $(1.42-3.11)$ & $<0,001$ \\
\hline Ventilation time $>48 \mathrm{~h}$ & 72 & (47 4) & 80 & $(14.6)$ & 14.49 & $(8.86-23.71)$ & $<0,001$ \\
\hline IABP perioperativ & 83 & $(47.4)$ & 92 & $(15.0)$ & & & $<0,001$ \\
\hline ECMO & 18 & $(10.3)$ & 157 & $(23.2)$ & & & $<0,001$ \\
\hline Blood transfusion & 156 & (97.6) & 4 & $(7.5)$ & 4.37 & $(1.55-12.30)$ & 0.001 \\
\hline$E K>5$ & 84 & $(48.3)$ & 90 & $(17.1)$ & 5.04 & $(3.44-7.37)$ & $<0,001$ \\
\hline Total blood loss >11 & 91 & $(62.8)$ & 52 & $(14.8)$ & 2.83 & $(1.93-4.14)$ & $<0,001$ \\
\hline Total blood loss > 21 & 38 & $(26.2)$ & 107 & $(18.8)$ & 4.43 & $(2.69-7.29)$ & $<0,001$ \\
\hline Dialysis & 27 & $(15.4)$ & 148 & (22.6) & 7.12 & $(3.58-14.14)$ & $<0,001$ \\
\hline Inotropie support $>48 \mathrm{~h}$ & 113 & $(77.4)$ & 33 & $(9.1)$ & 6.69 & $(4.35-10.28)$ & $<0,001$ \\
\hline CardioAssist & 105 & $(60.0)$ & 70 & (12.0) & 109.93 & $(49.16-245.83)$ & $<0,001$ \\
\hline $\mathrm{ACT}>150 \mathrm{sec}$ & 26 & $(21.1)$ & 97 & (21.3) & 1.69 & $(1.01-2.83)$ & 0.048 \\
\hline Pneumonia & 17 & (9.8) & 156 & (23.4) & 6.95 & $(2.94-16.41)$ & $<0,001$ \\
\hline Wound infection & 15 & (8.7) & 158 & $(25.0)$ & 1.02 & $(0.55-1.89)$ & 1.000 \\
\hline Operative re-exploration & 25 & $(14.5)$ & 148 & $(23.1)$ & 3.33 & $(1.86-5.97)$ & $<0,001$ \\
\hline
\end{tabular}

Pre-,intra- and postoperative data in univariaten analysis. The nominal variables are shown in absolute and percentage figures, cerebro vascular accident (CVA), hypertension (HT), 3 vessel-coronary artery disease (3 V-CAD), left main stenosis (LMS), perimyocardial infarction (PMI), red blood cells (RBC)

subgroup in order to provide the full analysis in patients with different risk profiles. Additionally, compared to the study by Warm Heart Investigators where cardioplegia was given antegradely and/or retrogradely depending on surgeon's preference this potential bias is not present in our study as cardioplegia was always administered antegradely [20]. Similar to our report, another study by Calafiore et al. investigated 500 consecutive CABG patients who received IWC compared to ICC [5]. However, the blood cardioplegia in that study was given antegradely in all cases, the main difference to our study is that Calafiore et al. only included elective CABG patients. Interestingly, Calafiore et al. found that the outcome in the IWC group was superior to the ICC group in terms of requirement for circulatory assistance, IABP, and incidence of myocardial infarction and cerebrovascular accidents. In another prospective randomized trial Franke et al. also showed a significantly improved myocardial protection in the IWC group compared to ICC group in elective setting [15]. Surprisingly, there is only one experimental animal study which showed that IWC is superior to ICC in ischemic myocardium [21]. Despite the fact that the concept of myocardial protection in the beginning of cardiac surgery was recommended with introduction of hypothermic crystalloid cardioplegia it has been continuously adjusted and improved by pharmacological additives, blood cardioplegia and different application techniques [22]. Despite the fact that warm and cold cardioplegia resulted in similar 30-days and long-term mortality in our study, there has been a substantial amount of research showing that warm cardioplegia reduces several adverse post-operative events $[7,23]$. However, totally different results were found in a meta-analysis with a total of 41 randomized controlled trials including 5879 patients comparing warm vs. cold cardioplegia. This study demonstrated no statistically significant differences in the incidences of clinical events [24]. Hence, our results seem to support this theory that there are no statistically significant differences in using cold vs. warm blood cardioplegia in emergent CABG patients particularly those with aortic cross clamp time less than 60 min according to the mean cross clamp time results of our study. Additionally, we performed a multivariate analysis which further showed that the type cardioplegia is not an independent predictor for PMI or 30-day mortality independent of the procedure urgency.

In terms of our additional findings suggesting preoperative risk factors for PMI the strongest independent predictor appeared to be preoperative use of antiplatelet drugs also supported by Micelli et al. demonstrated previously [25]. Our further findings on predictors of early mortality, such as decreased EF of less than $40 \%$, AF and PAD are also consistent with previous research [26, 27]. Whereas van Straten et al. found 
Table 6 Multivariate analysis, primary endpoint: 30-days mortality

\begin{tabular}{|c|c|c|c|}
\hline \multicolumn{4}{|c|}{ Multivariate analysis, primary endpoint: 30 -days mortality $(n=45)$} \\
\hline Variable & O.R. & $95 \%-C . I$. & $p$-value \\
\hline HLP & 0.39 & $(0.19-0.79)$ & 0.009 \\
\hline AF & 3.33 & $(1.49-7.47)$ & 0.003 \\
\hline $3 V-C A D$ & 0.36 & $(0.16-0.83)$ & 0.016 \\
\hline COPD & 0.26 & $(1.05-6.21)$ & 0.038 \\
\hline PAD & 2.51 & $(1.13-5.55)$ & 0.023 \\
\hline Statin & 0.38 & $(0.18-0.80)$ & 0.010 \\
\hline Antiplatelet. & 1.99 & $(0.95-4.17)$ & 0.070 \\
\hline$E F<40 \%$ & 3.66 & $(1.79-7.52)$ & $<0,001$ \\
\hline
\end{tabular}

Variable used in multivariable analysis:

Female

HT

pulm. HT

HLP

Diab. mellitus

AF

$3 \mathrm{~V}-\mathrm{CAD}$

LMS

COPD

$P A D$

Smoker

Crea $>2$

Renal insuff. preop Crea $<2$

CVA

Beta-blocker

Statin

Antiplatelet.

$\mathrm{EF}<40 \%$

IABP preop

AF Atrial fibrillation, CVA cerebrovascular accident, HLP Hyperlipidemia, $H T$ Hypertension, PAD peripherial artery disease, LMS left main stenosis

evidence that PAD is associated with an increased risk for long-term mortality, our findings suggested that PAD is also an independent predictor for short-term mortality [28]. Finally, our data support that preoperative statin treatment may have protective effects on mortality similarly to recent studies by Kulik et al. and Girerd et al. $[29,30]$ and prevents cardiovascular complications by pleiotropic effects [31]. Nevertheless, optimal mortality prediction should still be performed using established scoring systems $[32,33]$. Our study is also in consistence with the fact that statins may reduce cytokine release and neutrophil adhesion via a nitric oxide-mediated mechanism and improve postoperative myocardial perfusion of bypassed areas [34, 35].
Table 7 Multivariate analysis, endpoint: PMI

\begin{tabular}{llll}
\hline \multicolumn{4}{l}{ Multivariate analysis, endpoint: PMI $(n=175)$} \\
\hline Variable & O.R. & $95 \%$-C.I. & $p$-value \\
\hline AF & 1.79 & $(0.98-3.27)$ & 0.060 \\
CVA & 1.68 & $(0.98-2.89)$ & 0.059 \\
Statin & 0.66 & $(0.41-1.08)$ & 0.101 \\
Antiplatelet & 4.79 & $(3.10-7.40)$ & $<0,001$ \\
EF $<40 \%$ & 2.04 & $(1.32-3.15)$ & 0.001 \\
IABP preap & 3.68 & $(1.34-10.13)$ & 0.012 \\
Hemofiltration & 3.61 & $(2.22-5.87)$ & $<0,001$
\end{tabular}

Variable used in multivariable analysis:

art. HT

pulm. HT

AF

LMS

HLP

PAD

Crea $>2$

Renal insuff. preop Crea $<2$

CVA

Beta-blocker

Statin

Antiplatelet

$\mathrm{EF}<40 \%$

IABP preop.

OP-time after 08:00 pm

Hemofiltration

AF Atrial fibrillation, CVA cerebro vascular accident, HLP Hyperlipidemia, $H T$ Hypertension, PAD peripherial artery disease, LMS left main stenosis

\section{Conclusions}

Summarizing, there are no clinically significant differences in using cold and warm blood cardioplegia in emergent $\mathrm{CABG}$ patients including long-term outcomes regardless of the urgency status. Decreased EF, AF and PAD remain main predictors for 30-day mortalitiy whereas preoperative use of statins is associated with protective effects. Warm blood cardioplegia and normothermic systemic perfusion offer safe myocardial protection strategies in emergent CABG patients and IWC is a cost-effective alternative. Therefore, we suggest to use warm blood cardioplegia antegradely in elective and emergent CABG procedures regardless of the emergent status.

\section{Limitations}

This is a retrospective study with consecutive elective and emergent patients. Even though we consecutively matched the emergent cohort 1:2 to a control elective 
cohort to avoid selection bias there is still a limitation in interpreting the results due to the use of cardioplegia depending on surgeons' preferences. There were surgeons who routinely used cold blood cardioplegia and surgeons who routinely used warm blood cardioplegia which may have influenced cross clamp times in IWC and ICC groups.

\section{Competing interests}

The authors declare that they have no competing interests.

\section{Authors' contributions}

MZ (first author) created design of the study, performed data analysis and interpretation, and drafted the manuscript. AH (shared first author) participated in the design of the study, performed data management, statistical analysis and helped to draft the manuscript. PBR (shared first author) participated in the design of the study and performed the statistical analysis and contributed significantly in revising the manuscript. YHC participated in the design of the study, revised the manuscript critically and approved the final version. AS participated in the design of the study, calculated all statistics and revised the manuscript critically and approved the final version. MS participated in the study concept and data analysis, revised the manuscript and approved the final manuscript. AFP revised the manuscript and approved the final manuscript. AW participated in the design of the study and approved the final manuscript. AG participated in the design of the study and revised the manuscript and approved the final manuscript. ACD participated in the design of the study and approved the final manuscript. AK participated in the design of the study and approved the final manuscript. FKR approved the final manuscript. JW participated in the design of the study and approved the final manuscript. TW (senior author) approved the concept of the study, revised the article and approved the final version. All authors read and approved the final manuscript.

\section{Author details}

${ }^{1}$ Department of Cardiothoracic Surgery, Heart Center, University of Cologne, Kerpener Str. 62, 50937 Cologne, Germany. ${ }^{2}$ Department of Cardiothoracic Transplantation and Mechanical Circulatory Support, Royal Brompton \& Harefield NHS Foundation Trust, Harefield Hospital, Harefield, Middlesex, UK. ${ }^{3}$ Department of Cardiac Surgery, University of Heidelberg, Heidelberg, Germany. ${ }^{4}$ Heart and Vascular Institute, Pennstate Hershey, Philadelphia, PA, USA.

Received: 16 February 2015 Accepted: 18 November 2015 Published online: 04 December 2015

\section{References}

1. Bigelow WG, Lindsay WK, Greenwood WF. Hypothermia; its possible role in cardiac surgery: an investigation of factors governing survival in dogs at low body temperatures. Ann Surg. 1950;132:849-66.

2. Gay Jr WA, Ebert PA. Functional, metabolic, and morphologic effects of potassium-induced cardioplegia. Surgery. 1973;74:284-90.

3. Follette DM1, Steed DL, Foglia R, Fey K, Buckberg GD. Advantages of intermittent blood cardioplegia over intermittent ischemia during prolonged hypothermic aortic clamping. Circulation. 1978;58:1200-9.

4. Calafiore AM, Teodori G, Mezzetti A, Bosco G, Verna AM, Di Giammarco G, et al. Intermittent antegrade warm blood cardioplegia. Ann Thorac Surg. 1995; 59:398-402.

5. Calafiore AM, Teodori G, Di Giammarco G, Bosco G, Mezzetti A, Lapenna D, et al. Intermittent antegrade cardioplegia: warm blood vs cold crystalloid: a clinical study. J Cardiovasc Surg (Torino). 1994;35:179-84.

6. Guru V, Omura J, Alghamdi AA, Weisel R, Fremes SE. Is blood superior to crystalloid cardioplegia? A meta-analysis of randomized clinical trials. Circulation. 2006;114:1331-8.

7. Abah U, Garfjeld Roberts P, Ishaq M, De Silva R. Is cold or warm blood cardioplegia superior for myocardial protection? Interact Cardiovasc Thorac Surg. 2012;14:848-55.

8. Weymann A, Sabashnikov A, Patil NP, Konertz W, Modersohn D, Dohmen PM. Eprosartan improves cardiac function in swine working heart model of ischemia-reperfusion injury. Med Sci Monit Basic Res. 2014;20:55-62.
9. Liakopoulos OJ, Kuhn EW, Choi YH, Chang W, Wittwer T, Madershahian N, et al. Myocardial protection in cardiac surgery patients requiring prolonged aortic cross-clamp times: a single-center evaluation of clinical outcomes comparing two blood cardioplegic strategies. J Cardiovasc Surg (Torino). 2010;51:895-905.

10. Januzzi JL, Lewandrowski K, MacGillivray TE, Newell JB, Kathiresan S, Servoss $\mathrm{SJ}$, et al. A comparison of cardiac troponin T and creatine kinase-MB for patient evaluation after cardiac surgery. J Am Coll Cardiol. 2002;39:1518-23.

11. Mächler H, Gombotz H, Sabin K, Metzler H. Troponin T as a marker of perioperative myocardial cell damage. Adv Pharmacol. 1994;31:63-73.

12. Triggiani M, Simeone F, Gallorini C, Paolini G, Donatelli F, Paolillo G, et al. Measurement of cardiac troponin $T$ and myosin to detect perioperative myocardial damage during coronary surgery. Cardiovasc Surg. 1994;2:441-5.

13. Fremes SE, Christakis GT, Weisel RD, Mickle DA, Madonik MM, Ivanov J, et al. A clinical trial of blood and crystalloid cardioplegia. J Thorac Cardiovasc Surg. 1984;88:726-41.

14. Jasinski M, Kadzioła Z, Bachowski R, Domaradzki W, Wenzel-Jasinska I, Piekarski M, et al. Comparison of retrograde versus antegrade cold blood cardioplegia: randomized trial in elective coronary artery bypass patients. Eur J Cardiothorac Surg. 1997;12:620-6.

15. Franke UF, Korsch S, Wittwer T, Albes JM, Wippermann J, Kaluza M, et al. Intermittent antegrade warm myocardial protection compared to intermittent cold blood cardioplegia in elective coronary surgery-do we have to change? Eur J Cardiothorac Surg. 2003;23:341-6.

16. de Jonge M, van Boxtel AG, Soliman Hamad MA, Mokhles MM, Bramer S, Osnabrugge RL, et al. Intermittent warm blood versus cold crystalloid cardioplegia for myocardial protection: a propensity score-matched analysis of 12-year single-center experience. Perfusion 2014, doi:10.1177/ 0267659114540023

17. Dar Ml. Cold crystalloid versus warm blood cardioplegia for coronary artery bypass surgery. Ann Thorac Cardiovasc Surg. 2005;11:382-5.

18. Carrier M, Pelletier LC, Searle NR. Does retrograde administration of blood cardioplegia improve myocardial protection during first operation for coronary artery bypass grafting? Ann Thorac Surg. 1997;64:1256-61. discussion 1262.

19. Cakir H, Gur O, Ege T, Kunduracilar H, Ketenciler S, Duran E. Comparison of the efficacy of the cardiac hypothermia and normothermia to myocardial damage in coronary artery bypass graft surgery with systemic normothermic cardiopulmonary bypass. J Cardiovasc Surg (Torino). 2013;54:397-401.

20. Anonymous. Randomised trial of normothermic versus hypothermic coronary bypass surgery. The Warm Heart Investigators. Lancet. 1994:343:559-63.

21. Brown WM 3rd, Jay JL, Gott JP, Huang AH, Pan-Chih, Horsley WS, et al. Warm blood cardioplegia: superior protection after acute myocardial ischemia. Ann Thorac Surg. 1993;55:32-41. discussion 41-32.

22. Yamamoto $H$, Yamamoto F. Myocardial protection in cardiac surgery: a historical review from the beginning to the current topics. Gen Thorac Cardiovasc Surg. 2013;61:485-96.

23. Pelletier LC, Carrier M, Leclerc Y, Cartier R, Wesolowska E, Solymoss BC. Intermittent antegrade warm versus cold blood cardioplegia: a prospective, randomized study. Ann Thorac Surg. 1994;58:41-8. discussion 48-49.

24. Fan Y, Zhang AM, Xiao YB, Weng YG, Hetzer R. Warm versus cold cardioplegia for heart surgery: a meta-analysis. Eur J Cardiothorac Surg. 2010;37:912-9.

25. Miceli A, Duggan SM, Aresu G, de Siena PM, Romeo F, Glauber M, et al. Combined clopidogrel and aspirin treatment up to surgery increases the risk of postoperative myocardial infarction, blood loss and reoperation for bleeding in patients undergoing coronary artery bypass grafting. Eur J Cardiothorac Surg. 2013;43:722-8.

26. Hamad MA, van Straten AH, Schönberger JP, ter Woorst JF, de Wolf AM, Martens EJ, et al. Preoperative ejection fraction as a predictor of survival after coronary artery bypass grafting: comparison with a matched general population. J Cardiothorac Surg. 2010;5:29.

27. Soliman Hamad MA, van Straten AH, van Zundert AA, ter Woorst JF, Martens EJ, Penn OC. Preoperative prediction of early mortality in patients with low ejection fraction undergoing coronary artery bypass grafting. J Card Surg. 2011;26:9-15.

28. van Straten AH, Firanescu C, Soliman Hamad MA, Tan ME, ter Woorst JF, Martens EJ. Peripheral vascular disease as a predictor of survival after coronary artery bypass grafting: comparison with a matched general population. Ann Thorac Surg. 2010;89:414-20. 
29. Kulik A, Ruel M. Statins and coronary artery bypass graft surgery: preoperative and postoperative efficacy and safety. Expert Opin Drug Saf. 2009;8:559-71.

30. Girerd N, Pibarot P, Daleau P, Voisine P, O'Hara G, Després JP, et al. Statins reduce short- and long-term mortality associated with postoperative atrial fibrillation after coronary artery bypass grafting: impact of postoperative atrial fibrillation and statin therapy on survival. Clin Cardiol. 2012;35:430-6.

31. Schouten $\mathrm{O}, \mathrm{Bax} J$ J, Dunkelgrun $\mathrm{M}$, Feringa HH, van Urk H, Poldermans D. Statins for the prevention of perioperative cardiovascular complications in vascular surgery. J Vasc Surg. 2006:44:419-24.

32. Doerr F, Heldwein MB, Bayer O, Sabashnikov A, Weymann A, Dohmen PM, et al. Combination of European System for Cardiac Operative Risk Evaluation (EuroSCORE) and Cardiac Surgery Score (CASUS) to improve outcome prediction in cardiac surgery. Med Sci Monit Basic Res. 2015;21:172-8.

33. Doerr F, Heldwein MB, Bayer O, Sabashnikov A, Weymann A, Dohmen PM, et al. Inclusion of 'ICU-Day' in a logistic scoring system improves mortality prediction in cardiac surgery. Med Sci Monit Basic Res. 2015;21:145-52.

34. Liakopoulos OJ, Dörge H, Schmitto JD, Nagorsnik U, Grabedünkel J, Schoendube FA. Effects of preoperative statin therapy on cytokines after cardiac surgery. Thorac Cardiovasc Surg. 2006;54:250-4.

35. Dotani MI, Morise AP, Haque R, Jain AC, Gupta N, Gibson CM. Association between short-term simvastatin therapy before coronary artery bypass grafting and postoperative myocardial blood flow as assessed by positron emission tomography. Am J Cardiol. 2003;91:1107-9.

\section{Submit your next manuscript to BioMed Central and we will help you at every step:}

- We accept pre-submission inquiries

- Our selector tool helps you to find the most relevant journal

- We provide round the clock customer support

- Convenient online submission

- Thorough peer review

- Inclusion in PubMed and all major indexing services

- Maximum visibility for your research 